\title{
Comment on: Case report of ovarian torsion mimicking ovarian cancer as an uncommon late complication of laparoscopic supracervical hysterectomy
}

\author{
Artur Czekierdowski
}

Medical University, Lublin, Poland

Dear Editor,

I have read with interest the paper titled "Case report of ovarian torsion mimicking ovarian cancer as an uncommon late complication of laparoscopic supracervical hysterectomy" written by Ciebiera et al. and published in "Menopause Review" in 2016; 15: 223-226.

The authors described what they call an unusual presentation of an adnexal $+10 \mathrm{~cm}$ large mass with adnexal torsion in a 46-year-old woman who previously had laparoscopic hysterectomy with unilateral adnexectomy due to fibroids, and a haemorrhagic cyst of her left ovary. The patient had preoperative tumour markers assessment, pelvic ultrasound, and pelvic computed tomography. The Authors claim that all these studies indicated an "elevated risk of malignancy", and because of this, laparotomy with midline vertical incision was performed. During surgery they collected multiple cytological smears and "mid-surgical evaluation with the possibility of conversion to a full oncological profile (excision of the cervix, greater omentum, appendix, and lymphadenectomy)" was planned. To document their thesis, two preoperative sonographic images of the smooth-shaped solid-cystic mass are presented. Despite a detailed description of the preoperative diag-

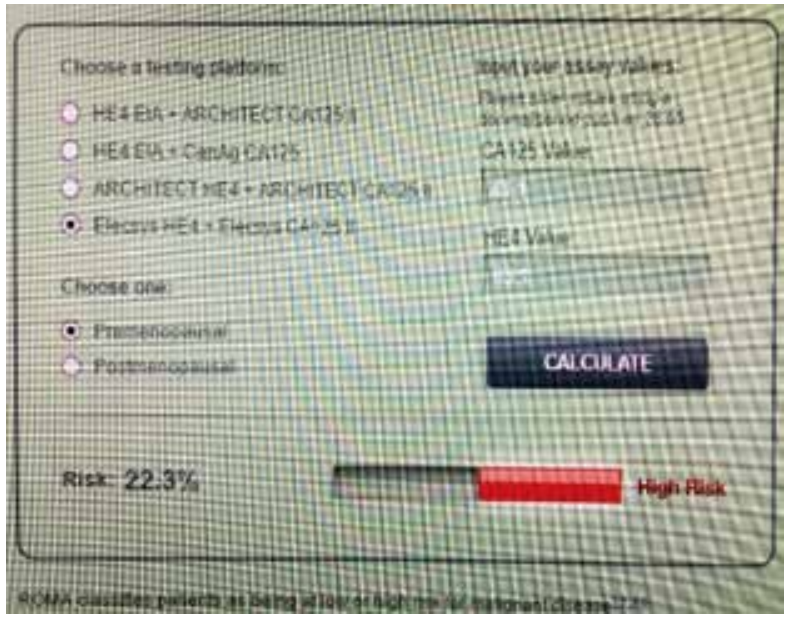

Fig. 1. Screenshot from the Risk of Ovarian Malignancy Algorithm online calculator nostic methods, there are a number of important issues around the design, analysis, and reporting of this case that I wish to raise.

First, ultrasound scans, contrary to the macroscopic picture of the removed tumour, are not presented in colour, so the vascularity of the mass is difficult/impossible to assess. Moreover, these "representative" scans were made in greyscale only, and because of this they do not contain a colour Doppler map on the right side of the images. Therefore, we have to believe the Author's claim that the subjective assessment of the examiner suggested high vascular content, at least in some portions of this mass. Secondly, since preoperative levels of serum CA-125 antigen and HE-4 protein were $41.1 \mathrm{U} / \mathrm{ml}$ and $83.1 \mathrm{pmol} / \mathrm{l}$, respectively, the Authors claim that the calculated Risk of Ovarian Malignancy Algorithm (ROMA) was $31.5 \%$, which, according to their beliefs, "classified the patient in the 'high risk for ovarian cancer' group". Unfortunately, this is not so easy. The patient, despite hysterectomy at the age of 46, was still premenopausal, because the menopause in women after uterus removal is stated as +50 years of age in most scientific papers. Premenopausal status makes a possibility of repeated haemorrhagic ovarian cyst more likely, and at the same time, the use of common serum ovarian tumour markers assessment much less reliable. According to e.g. the "he4test.com" website [1] containing an online calculator of ROMA, for this particular patient ROMA risk was equal to $22.3 \%$ (see attached Fig. 1).

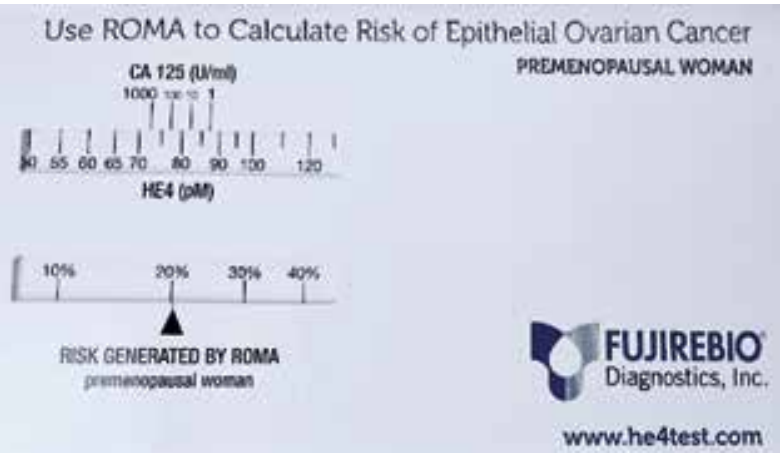

Fig. 2. ROMA premenopausal risk 


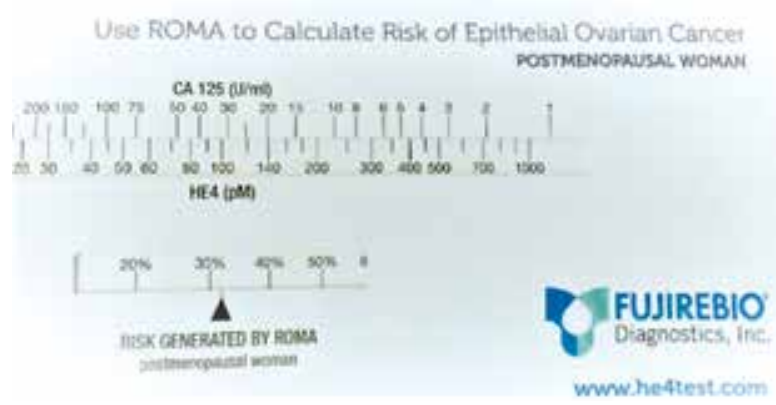

Fig. 3. ROMA postmenopausal risk

According to the Japanese based Fujirebio Company, which, along with the Abbott Company, USA, also manufactures HE4 tests and distributes ROMA risk manual calculators, the premenopausal woman with such tumour marker levels had a risk of app. 21-22\% (Fig. 2).

This value indicates only slightly elevated risk, strongly depending on the medical centre type (oncologic vs. non-oncologic) to which the patient was referred. According to the International Ovarian Tumour Analysis (IOTA) group studies, high risk of malignancy in gynaecological cancer centres, such as the Authors' hospital, should probably be set at 25-30\% [2]. Theoretically, for postmenopausal women the manually calculated ROMA risk in this case was app. 32\% (Fig. 3).

Thirdly, when diagnosis of a malignant ovarian mass is suspected, many recent studies have documented much better predictive values of preoperative sonography as compared to tumour markers or combined models, such as ROMA or RMI. For instance, in 2013 Kaijser et al. [2] concluded that the IOTA logistic regression model 2 (LR2), also used by the Authors in the presented case, shows better diagnostic performance than ROMA for the characterisation of a pelvic mass in both pre- and postmenopausal women. Also, in a recent meta-analysis Nunes et al. [3] found that simple rules protocol could be used in $76-89 \%$ of tumours, and in all analysed studies it was an accurate test for the diagnosis of ovarian cancer. Until last year, a second opinion by an ultrasound expert was required when this method in its classical version could not be applied [4]. In 2016 the IOTA group published a paper showing that the use of simple rules is possible in all cases of adnexal tumours, and the risk of malignancy of an adnexal mass can be assessed even without any calculator [5].

The problem with preoperative diagnosis of the presented case is related to the wrong use and misunderstanding of the IOTA group terms and definitions by the Authors. They have assigned tumour malignant feature M4 to an apparently regularly shaped solid-cystic mass. The International Ovarian Tumour Analysis definition of the M4 feature is straight-forward: "an irregular multilocular-solid tumour larger than 100 mm"[4]. However, the presented scans of their tumour contain only one

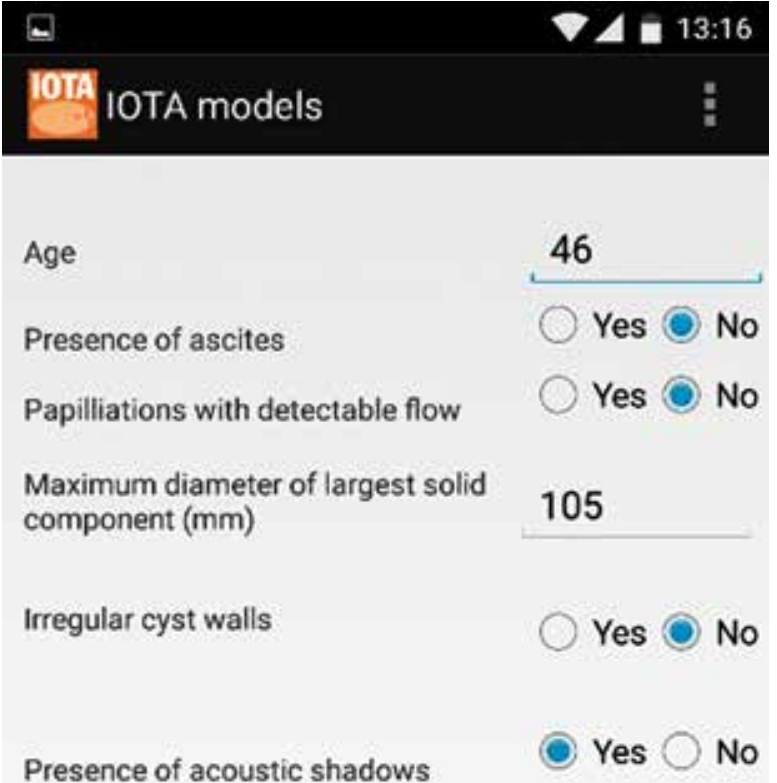

$3.88999999999999997 \%$

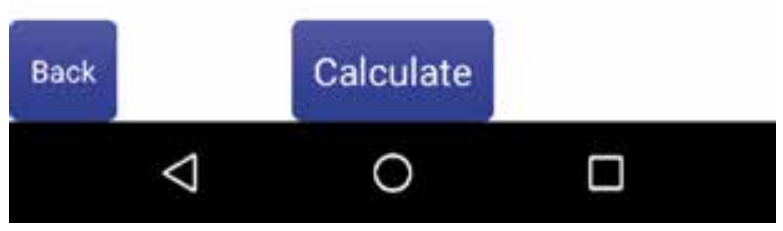

Fig. 4. Risk calculation in the IOTA logistic regression model 2, Android application

B feature, namely B3 which is "acoustic shadows". A similar situation has probably occurred with the Author's attempt to use the IOTA group LR2 model. Ciebiera et al. state that: "The LR2 IOTA model was also used and determined the risk for malignancy to be approximately $52 \%$ ". There is no explanation of which variables they have used and how exactly the calculation was done; was is on the website or mobile device or with the mathematical formula? In any event, the six variables of LR2 should be correctly scored as in Fig. 4.

The risk calculated by the model was only app. $3.89 \%$, which is very far from $50 \%$ as estimated by the Authors [2]. It seems that the presented case was completely wrongly assessed before the operation, and computed tomography scanning brought nothing new to the ultrasound diagnosis. Furthermore, a midline vertical incision laparotomy could be spared to this woman, with laparoscopy or minilaparotomy performed instead of a larger surgery. The reason for the wrong preoperative diagnosis seems to be obvious: it 
was a complete misunderstanding of the IOTA terms and definitions used to describe adnexal masses. It is not clear from the paper if any of the Authors of this case report has taken and passed the IOTA MCQ test for this reason, but even not having a relevant certificate should not prompt such a flawed opinion and decision.

Lastly, I would like to raise the question of the possibility of increased risk of adnexal torsion after laparoscopic supracervical hysterectomy. The Authors do not use any publications to support their thesis, nor can it be found in the medical literature. The main risk of laparoscopic hysterectomy is related to the morcellation of a uterine malignancy, and subsequent dissemination of occult cancer, or even uterine sarcoma. These dangerous complications, and probably not adnexal torsion, must be balanced by the risks of increased numbers of laparotomies.

In conclusion, I am happy to observe an attempt to use the IOTA models such as LR2 and, as recently suggested by the Polish Gynaecological Society Guidelines [2015], also Simple Rules for the trial of preoperative discrimination between benign and malignant tumour, as presented in this case report. However, the Author's analysis does not allow the reader of this paper to draw any reliable conclusions with respect to such benign and acute mass discrimination. Both ultrasound examiners and gynaecological surgeons must be aware that early diagnosis and appropriate surgical management of adnexal torsion is the only way to prevent complications like necrosis of the ovary and, in women planning pregnancy, to preserve their future fertility. To improve reporting of ultrasound-based prediction model results of various tumours including adnexal torsion, it is extremely important to be aware of the IOTA definitions and terms. This in turn may assist in the correct preoperative risk estimation and diagnosis of these patients.

Sincerely,

Prof. Artur Czekierdowski, MD, PhD IOTA Group member since 2006

\section{References}

1. http://romatools.he4test.com/calculator_row_en.html (access 2017.02.15).

2. Kaijser J, Van Gorp T, Van Hoorde K, et al. A comparison between an ultrasound based prediction model (LR2) and the risk of ovarian malignancy algorithm (ROMA) to assess the risk of malignancy in women with an adnexal mass. Gynecol Oncol 2013; 129: 377-383.

3. Nunes N, Ambler G, Foo X, et al. Use of IOTA simple rules for diagnosis of ovarian cancer: meta-analysis. Ultrasound Obstet Gynecol 2014; 44: 503-514.

4. Timmerman D, Testa AC, Bourne T, et al. Simple ultrasound-based rules for the diagnosis of ovarian cancer. Ultrasound Obstet Gynecol 2008; 31: 681-690.

5. Timmerman D, Van Calster B, Testa A, et al. Predicting the risk of malignancy in adnexal masses based on the Simple Rules from the International Ovarian Tumor Analysis group. Am J Obstet Gynecol 2016; 214: 424-437. 